\title{
月地高速再入返回飞行器陀螺在轨自主标定技术 研究及实现
}

\author{
于丹 ${ }^{(1 *}$, 董文强 ${ }^{(1)}$, 王勇 ${ }^{(1)}$, 郑永洁 ${ }^{(1)}$, 杨鸣 ${ }^{(1)}$, 张㻇 ${ }^{(1)}$, 张国锋 ${ }^{(1}$, 王志毅 ${ }^{(1)}$ \\ (1) 北京控制工程研究所, 北京 100190 ; \\ (2) 空间智能控制技术重点实验室，北京 100190 \\ *E-mail: 1mtberyu@ vip.sina.com.cn
}

收稿日期: 2014-12-08; 接受日期: 2014-12-28

国家中长期科技发展规划重大专项资助项目

\begin{abstract}
摘要针对包含非正交安装陀螺的捷联惯导平台轻量化自主对准问题, 建立了非 正交陀螺组误差模型, 提出一套基于最小二乘法的标定算法和地面试验验证方法, 在计算能力受限的情况下完成了高精度在轨标定. 地面数学仿真和试验验证结果表 明，该方法与扩展卡尔曼滤波法精度相当，计算量却大幅减小。在此基础上，通过对 标定算法进行逻辑设计, 实现了在轨多组陀螺同步容错和标定. 该方法已成功应用 于探月工程三期月地高速再入返回飞行器的实际飞行, 实现了 2 套惯性导航平台的 高精度初始姿态同步对准.
\end{abstract}

\section{关键词}

探月三期

月地高速再入返回飞行器 惯性测量单元

轻量化

自主标定

\section{1 引言}

月地高速再入返回飞行器的基本导航手段是惯 性导航，导航开始前需要确定初始基准，包括对惯性 测量单元(IMU)的标定, 以及位置、速度和姿态初值 的确定. 对于空间飞行器, 通常采用星敏感器和陀螺 滤波的方式, 实现对陀螺常值漂移的标定, 但由于飞 行器上的星敏感器与陀螺安装于不同舱段, 安装偏 差会因发射振动及在轨结构热变形等因素发生变化, 进而导致捷联惯导的数学平台偏斜, 从而影响导航 精度, 因此, 在飞行器再入返回过程导航建立前需对 惯导系统进行传递对准.

传统惯性平台基座传递对准的研究主要集中在 模型、算法、试验评估三个方面. 失准角误差模型主
要有 $\Psi$ 角误差模型 ${ }^{[1]} 、 \Phi$ 角误差模型 ${ }^{[2]}$ 、量测失准角模 型 ${ }^{[3]}$, 文献[4]给出了惯导系统正交安装误差模型的 统一方法, 将现有的各种误差模型纳入到统一的框 架之中. 传统对准算法基于测量/计算参数匹配+滤波 实现 ${ }^{[5]}$, 在 Kain 提出速度+姿态匹配法后, 发展出“速 度+姿态匹配”、“速度+姿态变化量匹配”等多种匹配方

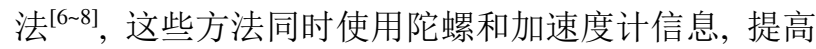
了初始对准的快速性和准确度, 但由于空间飞行器在 轨无法实施大过载机动, 且陀螺组可能存在非正交的 安装情况，上述方法均不能满足空间飞行器对导航平 台中陀螺安装偏差的高精度快速标定需求 ${ }^{[9]}$.

国际上进行类似任务的飞行器鲜见报道，从已 经公开的资料看, 美国的卡西尼探测器进行了类似 的导航平台对准, 但其数据处理是在地面完成的, 而

引用格式: 于丹, 董文强, 王勇, 等. 月地高速再入返回飞行器陀螺在轨自主标定技术研究及实现. 中国科学: 技术科学, 2015, 45: 213-220 Yu D, Dong W Q, Wang Y, et al. Research and implementation of on-orbit self-calibration for gyroscope of circumlunar return and reentry spacecraft (in Chinese). Sci Sin Tech, 2015, 45: 213-220, doi: 10.1360/N092014-00481 
非在轨自主计算 ${ }^{[10]}$. 嫦娥三号探测器结合自身任务 特点, 在常规卫星采用的星敏感器和陀螺滤波算法 的基础上, 研究了一套两层滤波算法. 第一层算法为 第二层算法提供较为准确的星体姿态角速度估值; 第二层算法将陀螺刻度因子误差, 安装误差和常值 漂移均作为滤波状态量, 以星敏感器作为外部姿态 信息来源, 使用 UD 分解的卡尔曼滤波器进行状态估 计, 并设计了适当的角速度机动以提高滤波器的可 观性 ${ }^{[11]}$. 但对于月地高速再入返回飞行器来讲, 受飞 行程序和测控弧段的约束, 导致导航平台对准的时 间有限，且在系统配置上采用不同体制的 IMU 形成 异构备份, 要求在无故障情况下具有对 2 套正交 IMU 组同时进行对准的基础上, 还需确保在发生故障情 况下, 重构后具备对非正交 IMU 组同时进行对准的 能力. 嫦娥三号探测器上采用的双层滤波算法中, 扩 展卡尔曼滤波达到了 15 维, 器载计算机无法同时完 成多套 15 维的扩展卡曼滤波算法的在线计算. 与此 同时, 发展出了多种数学仿真和半物理试验验证系 统, 在传递对准的地面试验研究中, Jones 等人 ${ }^{[12]}$ 设 计了包括机动运动模块、载体动力学模块等 11 个模 块在内的数学仿真环境, Shortelle 等人 ${ }^{[13]}$ 使用 F-16飞 机进行物理试验, 常见的试验系统还有跑车试验系 统 ${ }^{[14]}$, 但能够在试验室内进行有效验证的高精度物 理试验方法较为欠缺.

根据上述任务需求, 本文设计了一种基于最小 二乘法的轻量化在轨陀螺标定算法和地面试验验证 方法, 能够在有限计算能力下同时多组陀螺进行在 轨标定. 本方法具有对准精度高, 容错能力强, 故障 数据易于剔除的特点.

\section{2 陀螺安装误差的标定算法研究}

\section{1 非正交陀螺组误差模型}

文献[11]给出了 3 个接近正交陀螺的标定算法的 推导过程. 从推导过程上看, 因为使用了小角度近似 和接近正交情况下的陀螺刻度因子误差计算公式, 所以当 3 个陀螺正交性较差时, 存在刻度因子和安装 偏差估计不准的问题. 本文从非正交陀螺组的误差 模型出发, 不使用正交性假设条件, 得到了一组新的 陀螺刻度因子和安装偏差标定公式, 将标定算法推 广到了非正交安装的陀螺组.
假设陀螺组由 3 个非共面的陀螺构成(3 个陀螺 可能正交, 也可能不正交), 预先装订的 3 个陀螺的安 装矢量为 $\overrightarrow{\boldsymbol{G}}_{1}, \overrightarrow{\boldsymbol{G}}_{2}$ 和 $\overrightarrow{\boldsymbol{G}}_{3}, 3$ 个陀螺敏感轴的实际指向 矢量为 $\overrightarrow{p_{G 1}}, \overrightarrow{p_{G 2}}, \overrightarrow{p_{G 3}}$, 记 3 个陀螺安装的偏差矢量 为 $\Delta \overrightarrow{\boldsymbol{G}}_{1}, \Delta \overrightarrow{\boldsymbol{G}}_{2}$ 和 $\Delta \overrightarrow{\boldsymbol{G}}_{3}$.

实际的陀螺敏感轴矢量 $\overrightarrow{\boldsymbol{p}_{G 1}}$ 可以表达为

$$
\overrightarrow{\boldsymbol{p}_{G 1}}=\overrightarrow{\boldsymbol{G}}_{1}+\Delta \overrightarrow{\boldsymbol{G}}_{1},
$$

其中 $\Delta \overrightarrow{\boldsymbol{G}}_{1}$ 由装订的陀螺敏感轴的 3 个矢量表达为

$$
\Delta \overrightarrow{\boldsymbol{G}}_{1}=\Delta_{\boldsymbol{G} 11}^{\prime} \overrightarrow{\boldsymbol{G}}_{1}+\Delta_{\boldsymbol{G} 12}^{\prime} \overrightarrow{\boldsymbol{G}}_{2}+\Delta_{\boldsymbol{G} 13}^{\prime} \overrightarrow{\boldsymbol{G}}_{3} .
$$

通过上述推导得出, 陀螺的实际敏感轴矢量与 已装订的陀螺敏感轴矢量线性组合关系:

$$
\overrightarrow{\boldsymbol{p}_{\boldsymbol{G} 1}}=\left(1+\Delta_{G 11}^{\prime}\right) \overrightarrow{\boldsymbol{G}}_{1}+\Delta_{G 12}^{\prime} \overrightarrow{\boldsymbol{G}}_{2}+\Delta_{G 13}^{\prime} \overrightarrow{\boldsymbol{G}}_{3},
$$

即 $\overrightarrow{\boldsymbol{p}_{\boldsymbol{G}}}$ 在 $\overrightarrow{\boldsymbol{G}}_{1}, \overrightarrow{\boldsymbol{G}}_{2}$ 和 $\overrightarrow{\boldsymbol{G}}_{3}$ 组成的坐标系(不要求为直角 坐标系)中的坐标为

$$
p_{G 1}^{G}=\left[\begin{array}{c}
1+\Delta_{G 11}^{\prime} \\
\Delta_{G 12}^{\prime} \\
\Delta_{G 13}^{\prime}
\end{array}\right],
$$

这里 $\Delta_{G 11}, \Delta_{G 12}$ 和 $\Delta_{G 13}$ 是 3 个小偏差量. 同样 $\overrightarrow{\boldsymbol{p}_{G 2}}$ 和 $\overrightarrow{p_{G 3}}$ 在陀螺装订敏感轴矢量组成的坐标系中的表达为

$$
p_{G 2}^{G}=\left[\begin{array}{c}
\Delta_{G 21}^{\prime} \\
1+\Delta_{G 22}^{\prime} \\
\Delta_{G 23}^{\prime}
\end{array}\right],
$$

和

$$
\boldsymbol{p}_{G 3}^{G}=\left[\begin{array}{c}
\Delta_{G 31}^{\prime} \\
\Delta_{G 232}^{\prime} \\
1+\Delta_{G 33}^{\prime}
\end{array}\right] .
$$

令 $\overline{\boldsymbol{M}}_{b}^{G}$ 表示预先装订的陀螺安装矩阵, 其各行

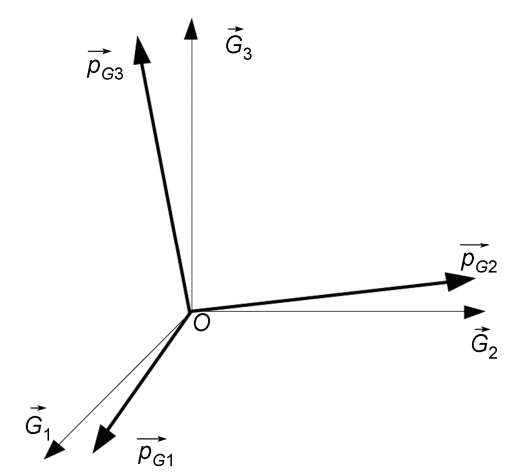

图 1 IMU 平台偏差示意图 
对应陀螺的安装向量, 则陀螺实际敏感轴在飞行器 本体坐标系下的坐标为

$$
\begin{aligned}
& \boldsymbol{p}_{G 1}^{b}=\left(\overline{\boldsymbol{M}}_{b}^{G}\right)^{-1}\left[\begin{array}{c}
1+\Delta_{G 11}^{\prime} \\
\Delta_{G 12}^{\prime} \\
\Delta_{G 13}^{\prime}
\end{array}\right], \\
& \boldsymbol{p}_{\boldsymbol{G} 2}^{b}=\left(\overline{\boldsymbol{M}}_{b}^{G}\right)^{-1}\left[\begin{array}{c}
\Delta_{G 21}^{\prime} \\
1+\Delta_{G 22}^{\prime} \\
\Delta_{G 23}^{\prime}
\end{array}\right],
\end{aligned}
$$

$$
\boldsymbol{p}_{\boldsymbol{G} 3}^{b}=\left(\overline{\boldsymbol{M}}_{b}^{G}\right)^{-1}\left[\begin{array}{c}
\Delta_{\boldsymbol{G} 31}^{\prime} \\
\Delta_{\boldsymbol{G} 32}^{\prime} \\
1+\Delta_{\boldsymbol{G} 33}^{\prime}
\end{array}\right] .
$$

设飞行器本体系下的角速度为 $\boldsymbol{\omega}^{b}$, 在 3 个陀螺 敏轴的投影为

$$
\boldsymbol{\omega}_{G i}=\left(\boldsymbol{p}_{G i}^{b}\right)^{\mathrm{T}} \boldsymbol{\omega}^{b}(i=1,2,3) .
$$

考虑陀螺的刻度因子误差 $\Delta K_{G}$, 常值漂移 $b_{G i}$ 和 测量噪声 $n_{G}$, 陀螺的测量值 $\tilde{\boldsymbol{\omega}}_{G}$ 可表示为

$$
\tilde{\omega}_{G i}=\left(1+\Delta K_{G i}\right) \omega_{G i}+b_{G i}+n_{G i}(i=1,2,3) .
$$

由陀螺测量值计算的飞行器三轴角速度 $\tilde{\boldsymbol{\omega}}^{b}$ 为

$$
\begin{aligned}
\tilde{\boldsymbol{\omega}}^{b} & =\left(\overline{\boldsymbol{M}}_{b}^{G}\right)^{-1}\left[\begin{array}{ccc}
\left(1+\Delta K_{G 1}\right)\left(1+\Delta_{G 11}^{\prime}\right) & \left(1+\Delta K_{G 1}\right) \Delta_{G 12}^{\prime} & \left(1+\Delta K_{G 1}\right) \Delta_{G 13}^{\prime} \\
\left(1+\Delta K_{G 2}\right) \Delta_{G 21}^{\prime} & \left(1+\Delta K_{G 2}\right)\left(1+\Delta_{G 22}^{\prime}\right) & \left(1+\Delta K_{G 2}\right) \Delta_{G 23}^{\prime} \\
\left(1+\Delta K_{G 3}\right) \Delta_{G 31}^{\prime} & \left(1+\Delta K_{G 3}\right) \Delta_{G 32}^{\prime} & \left(1+\Delta K_{G 3}\right)\left(1+\Delta_{G 33}^{\prime}\right)
\end{array}\right] \overline{\boldsymbol{M}}_{b}^{G} \boldsymbol{\omega}^{b} \\
& +\left(\overline{\boldsymbol{M}}_{b}^{G}\right)^{-1}\left[\begin{array}{c}
b_{G 1}+n_{G 1} \\
b_{G 2}+n_{G 2} \\
b_{G 3}+n_{G 3}
\end{array}\right] .
\end{aligned}
$$

得到陀螺角速度测量误差 $\delta \boldsymbol{\omega}^{b}$

$$
\begin{aligned}
\delta \boldsymbol{\omega}^{b} & =\tilde{\boldsymbol{\omega}}^{b}-\boldsymbol{\omega}^{b} \\
& =\left(\overline{\boldsymbol{M}}_{b}^{G}\right)^{-1}\left[\begin{array}{ccc}
\left(1+\Delta K_{G 1}\right)\left(1+\Delta_{G 11}^{\prime}\right)-1 & \left(1+\Delta K_{G 1}\right) \Delta_{G 12}^{\prime} & \left(1+\Delta K_{G 1}\right) \Delta_{G 13}^{\prime} \\
\left(1+\Delta K_{G 2}\right) \Delta_{G 21}^{\prime} & \left(1+\Delta K_{G 2}\right)\left(1+\Delta_{G 22}^{\prime}\right)-1 & \left(1+\Delta K_{G 2}\right) \Delta_{G 23}^{\prime} \\
\left(1+\Delta K_{G 3}\right) \Delta_{G 31}^{\prime} & \left(1+\Delta K_{G 3}\right) \Delta_{G 32}^{\prime} & \left(1+\Delta K_{G 3}\right)\left(1+\Delta_{G 33}^{\prime}\right)-1
\end{array}\right] \overline{\boldsymbol{M}}_{b}^{G} \boldsymbol{\omega}^{b} \\
& +\left(\overline{\boldsymbol{M}}_{b}^{G}\right)^{-1}\left[\begin{array}{c}
b_{G 1}+n_{G 1} \\
b_{G 2}+n_{G 2} \\
b_{G 3}+n_{G 3}
\end{array}\right] .
\end{aligned}
$$

进一步推导得到

$$
\begin{aligned}
\overline{\boldsymbol{M}}_{b}^{G} \delta \boldsymbol{\omega}^{b} & =\left[\begin{array}{ccc}
\left(1+\Delta K_{G 1}\right)\left(1+\Delta_{G 11}^{\prime}\right)-1 & \left(1+\Delta K_{G 1}\right) \Delta_{G 12}^{\prime} & \left(1+\Delta K_{G 1}\right) \Delta_{G 13}^{\prime} \\
\left(1+\Delta K_{G 2}\right) \Delta_{G 21}^{\prime} & \left(1+\Delta K_{G 2}\right)\left(1+\Delta_{G 22}^{\prime}\right)-1 & \left(1+\Delta K_{G 2}\right) \Delta_{G 23}^{\prime} \\
\left(1+\Delta K_{G 3}\right) \Delta_{G 31}^{\prime} & \left(1+\Delta K_{G 3}\right) \Delta_{G 32}^{\prime} & \left(1+\Delta K_{G 3}\right)\left(1+\Delta_{G 33}^{\prime}\right)-1
\end{array}\right] \overline{\boldsymbol{M}}_{b}^{G} \boldsymbol{\omega}^{b} \\
& +\left[\begin{array}{c}
b_{G 1}+n_{G 1} \\
b_{G 2}+n_{G 2} \\
b_{G 3}+n_{G 3}
\end{array}\right] .
\end{aligned}
$$

其中 $\overline{\boldsymbol{M}}_{b}^{G} \delta \boldsymbol{\omega}^{b}$ 是星敏和陀螺滤波得到的三轴陀螺漂移 再分解到各陀螺轴头上的等效漂移量, 可通过文献 [11]中所列的星敏+陀螺滤波算法得到. 由于采用匀 速转动策略, 在角速度控制稳定度较高的条件下, 在 滤波收玫后, 陀螺等效漂移量理论上为常值, 可通过
一段时间的统计得到均值, 记为 $\left[\begin{array}{lll}\hat{b}_{G 1}^{\prime} & \hat{b}_{G 2}^{\prime} & \hat{b}_{G 3}^{\prime}\end{array}\right]^{\mathrm{T}}$.

\section{2 基于最小二乘的标定误差算法}

多次绕 3 个不同本体轴进行匀速转动, 根据(14) 式可得到 


$$
\begin{gathered}
{\left[\begin{array}{cc}
\left(\overline{\boldsymbol{M}}_{b}^{G} \boldsymbol{\omega}_{1}^{b}\right)^{\mathrm{T}} & 1 \\
\left(\overline{\boldsymbol{M}}_{b}^{G} \boldsymbol{\omega}_{2}^{b}\right)^{\mathrm{T}} & 1 \\
\vdots & \vdots \\
\left(\overline{\boldsymbol{M}}_{b}^{G} \boldsymbol{\omega}_{n}^{b}\right)^{\mathrm{T}} & 1
\end{array}\right]\left[\begin{array}{c}
\left(1+\Delta K_{G 1}\right)\left(1+\Delta_{G 11}^{\prime}\right)-1 \\
\left(1+\Delta K_{G 1}\right) \Delta_{G 12}^{\prime} \\
\left(1+\Delta K_{G 1}\right) \Delta_{G 13}^{\prime} \\
b_{G 1}
\end{array}\right]=\left[\begin{array}{c}
\hat{b}_{G 11}^{\prime} \\
\hat{b}_{G 12}^{\prime} \\
\vdots \\
\hat{b}_{G 1 n}^{\prime}
\end{array}\right],} \\
{\left[\begin{array}{cc}
\left(\overline{\boldsymbol{M}}_{b}^{G} \boldsymbol{\omega}_{1}^{b}\right)^{\mathrm{T}} & 1 \\
\left(\overline{\boldsymbol{M}}_{b}^{G} \boldsymbol{\omega}_{2}^{b}\right)^{\mathrm{T}} & 1 \\
\vdots & \vdots \\
\left(\overline{\boldsymbol{M}}_{b}^{G} \boldsymbol{\omega}_{n}^{b}\right)^{\mathrm{T}} & 1
\end{array}\right]\left[\begin{array}{c}
\left(1+\Delta K_{G 2}\right) \Delta_{G 21}^{\prime} \\
\left(1+\Delta K_{G 2}\right)\left(1+\Delta_{G 22}^{\prime}\right)-1 \\
\left(1+\Delta K_{G 2}\right) \Delta_{G 23}^{\prime} \\
b_{G 2}+n_{G 2}
\end{array}\right]=\left[\begin{array}{c}
\hat{b}_{G 21}^{\prime} \\
\hat{b}_{G 22}^{\prime} \\
\vdots \\
\hat{b}_{G 2 n}^{\prime}
\end{array}\right],} \\
{\left[\begin{array}{cc}
\left(\overline{\boldsymbol{M}}_{b}^{G} \boldsymbol{\omega}_{1}^{b}\right)^{\mathrm{T}} & 1 \\
\left(\overline{\boldsymbol{M}}_{b}^{G} \boldsymbol{\omega}_{2}^{b}\right)^{\mathrm{T}} & 1 \\
\vdots & \vdots \\
\left(\overline{\boldsymbol{M}}_{b}^{G} \boldsymbol{\omega}_{n}^{b}\right)^{\mathrm{T}} & 1
\end{array}\right]\left[\begin{array}{c}
\left(1+\Delta K_{G 3}\right) \Delta_{G 31}^{\prime} \\
\left(1+\Delta K_{G 3}\right) \Delta_{G 32}^{\prime} \\
\left(1+\Delta K_{G 3}\right)\left(1+\Delta_{G 33}^{\prime}\right)-1 \\
b_{G 3}
\end{array}\right]=\left[\begin{array}{c}
\hat{b}_{G 31}^{\prime} \\
\hat{b}_{G 32}^{\prime} \\
\vdots \\
\hat{b}_{G 3 n}^{\prime}
\end{array}\right] .}
\end{gathered}
$$

令

$$
\boldsymbol{A}=\left[\begin{array}{cc}
\left(\overline{\boldsymbol{M}}_{b}^{G} \boldsymbol{\omega}_{1}^{b}\right)^{\mathrm{T}} & 1 \\
\left(\overline{\boldsymbol{M}}_{b}^{G} \boldsymbol{\omega}_{2}^{b}\right)^{\mathrm{T}} & 1 \\
\vdots & \vdots \\
\left(\overline{\boldsymbol{M}}_{b}^{G} \boldsymbol{\omega}_{n}^{b}\right)^{\mathrm{T}} & 1
\end{array}\right],
$$

选择转动顺序使得 $\boldsymbol{A}$ 为列满秩, 便可通过最小二乘 法获得各个偏差项.

\section{3 轻量化设计方法}

文献[11]采用 15 维的扩展卡尔曼滤波算法对姿 态, 刻度因子误差, 陀螺常漂, 安装偏差进行标定, 为了保证系统的可观性, 不能使用简化的常滤波系 数, 因此需要每个计算周期都解算 Raccati 方程, 为 了保证滤波的稳定性, 飞行器上计算机采用基于 UD 分解的扩展卡尔曼滤波，其占用的计算时间仍超出 了控制周期的一半. 月地高速再入返回飞行器上受 计算能力与测控弧段限制, 无法采用扩展卡尔曼滤 波同时对 2 套陀螺进行标定. 本文所提出的基于最小 二乘的标定计算方法, 每个控制周期只进行少量的 陀螺等效常值漂移统计计算, 其每个周期的计算量 远小于扩展卡尔曼滤波, 在标定转动序列结束后, 按 照最小二乘的方式解算各误差项, 计算量达到了轻 量化的要求.

\section{4 非正交陀螺组同步在轨标定策略及容错方法}

基于 2.3 节的轻量化设计, 使得有能力在轨同时 完成两组陀螺进行标定. 而且当有一个或多个陀螺
发生故障的情况下能够完成标定. 标定期间的软件 结构如图 2 所示, 具体步骤如下:

1) 用第一组角度增量计算飞行器四元数 $\boldsymbol{q}$. 采 用第一组陀螺的脉冲数和第一组估计的陀螺常漂计 算计算四元数, 对于故障情况, 从第一组 6 个陀螺中 选择出 3 个.

2) 用第二组角度增量计算飞行器四元数 $\boldsymbol{q}^{(2)}$. 采 用第二组陀螺的脉冲数和第二组估计的陀螺常漂计 算四元数. 对于故障情况, 从第二组 6 个陀螺中选择 出 2 个.

3) 根据故障诊断结果从 $\boldsymbol{q}$ 和 $\boldsymbol{q}^{(2)}$ 里选用一组四元 数作为 $\boldsymbol{q}^{\text {use }}$, 对星敏感器数据进行有效性检验.

4) 采用星敏感器新息对第一组 $\boldsymbol{q}$ 和陀螺常值漂 移进行修正, 并统计第一组陀螺的常值漂移均值.

5) 采用星敏感器新息对第二组 $\boldsymbol{q}^{(2)}$ 和陀螺常值 漂移进行修正, 并统计第二组陀螺的常值漂移均值.

6) 在标定机动序列完成 $10 \mathrm{~s}$ 后, 计算修正量.

在正常情况下第一组陀螺中只包含第一套 IMU 中的 3 个陀螺, 第二组陀螺中只包含第二套 IMU 中 的 3 个陀螺. 当某一个陀螺故障, 如第二套 IMU 中某 一个陀螺故障时, 第二组陀螺中包含第二套 IMU 中 的 2 个陀螺和第一套 IMU 中的 1 个陀螺, 第一组陀 螺仍然只包含第一套 IMU 中的 3 个陀螺, 这时第一 套 IMU 中有一陀螺参加了 2 个标定组, 其标定结果

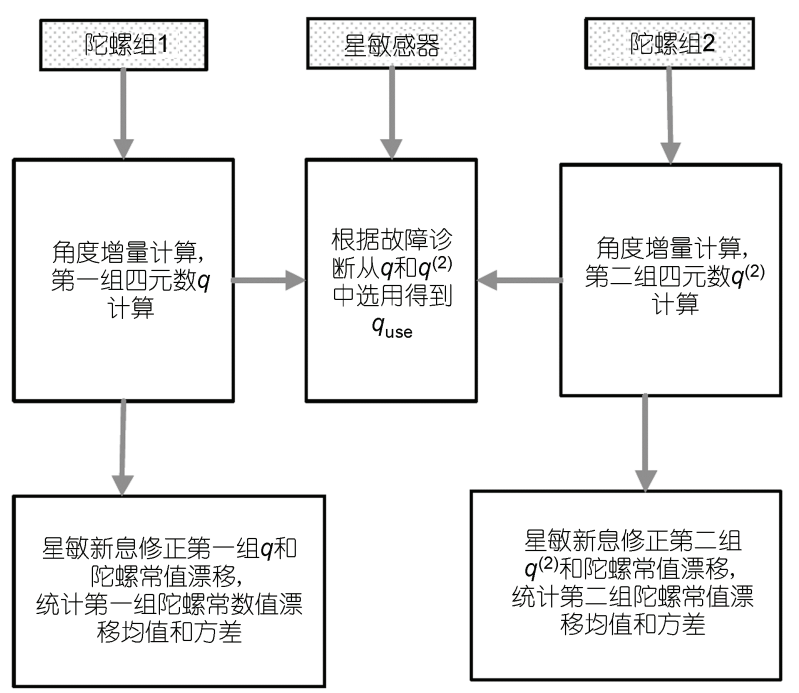

机动完成后, 用第一组和第二组统计的常值漂移均值计算安 装偏差、刻度系数和常值漂移

图 2 IMU 在轨标定软件结构 
优先选用第一标定组的结果, 同时第二套 IMU 中的 2 个正常陀螺也完成了在轨标定, 不会因为该套 IMU 中有 1 个陀螺故障而不能标定.

\section{3 地面验证及飞行结果评价}

\section{1 在轨算法的数学仿真分析}

通过对正交陀螺组和非正交陀螺组, 存在 $1^{\circ}$ 以 内的陀螺安装偏差进行 1000 组打靶仿真统计. 仿真 结果表明: 在轨标定可以有效减少陀螺安装误差、刻 度因子和陀螺常值漂移不确定性, 安装偏差标定精 度优于 $0.01^{\circ}$, 刻度因子误差标定精度优于 $1 \times 10^{-4}$, 陀螺常值漂移标定精度优于 $0.01 \% \mathrm{~h}$, 系统故障诊断 和容错策略正确.

\section{2 地面综合验证结果}

月地高速再入返回飞行器在轨飞行前, 在实验 室中采用等效星光模拟和陀螺激励的方式, 进行了 实物验证. 分别采用最小二乘和扩展卡尔曼滤波算 法, 设置陀螺安装方向偏差, 验证 3 个陀螺的标定精 度, 从表 5 和 6 中可以看到, 2 种算法标定精度相当.

进一步, 采用转台模拟在轨实际标定动态转动 过程, 进行半物理仿真. 按照飞行器上 IMU 构型安 装在三轴转台上. 外部光学标定系统基于经纬仪实 现, 通过地理基准平面镜、转台载物台上的立方镜以

表 1 正交组 IMU 标定前偏差

\begin{tabular}{ccccc}
\hline 组别 & 陀螺标号 & 安装偏差 $\left(^{\circ}\right)$ & $\begin{array}{c}\text { 刻度因 } \\
\text { 子误差 }\end{array}$ & $\begin{array}{c}\text { 陀螺常值漂移 } \\
\left({ }^{\circ} / \mathrm{h}\right)\end{array}$ \\
\hline 标定组 1 & 1 & 0.7071 & 0.001 & 9 \\
标定组 1 & 2 & 0.7071 & -0.001 & 11 \\
标定组 1 & 3 & 0.7102 & 0.0005 & 0.1 \\
标定组 2 & 4 & 0.8165 & 0.0001 & 7 \\
标定组 2 & 5 & 0.3608 & -0.001 & 7 \\
标定组 2 & 6 & 0.8411 & 0.0005 & 10 \\
\hline
\end{tabular}

\section{表 2 正交组 IMU 标定后残差}

\begin{tabular}{ccccc}
\hline 组别 & 陀螺标号 & 安装偏差 $\left({ }^{\circ}\right)$ & $\begin{array}{c}\text { 刻度因子 } \\
\text { 估计误差 }\end{array}$ & $\begin{array}{c}\text { 陀螺常值漂移 } \\
\text { 估计误差 } \\
(\% / h)\end{array}$ \\
\hline 标定组 1 & 1 & 0.0041 & 0.000028 & 0.0067 \\
标定组 1 & 2 & 0.0028 & 0.000009 & -0.0045 \\
标定组 1 & 3 & 0.0012 & -0.000042 & 0.00521 \\
标定组 2 & 4 & 0.0026 & -0.000034 & 0.0121 \\
标定组 2 & 5 & 0.0043 & -0.000022 & -0.0082 \\
标定组 2 & 6 & 0.0059 & 0.000036 & -0.0073 \\
\hline
\end{tabular}

\section{表 3 非正交组 IMU 标定前偏差}

\begin{tabular}{|c|c|c|c|c|}
\hline 组别 & 陀螺标号 & 安装偏差 $\left(^{\circ}\right)$ & 刻度因子误 & $\begin{array}{l}\text { 常值漂移 } \\
(\% / \mathrm{h})\end{array}$ \\
\hline 标定组 1 & 1 & 0.7071 & 0.0001 & 0.2 \\
\hline 标定组 1 & 3 & 0.7102 & 0.0005 & 0.1 \\
\hline 标定组 1 & 4 & 0.8165 & 0.001 & 1 \\
\hline 标定组 2 & 2 & 0.7071 & -0.001 & 0.3 \\
\hline 标定组 2 & 5 & 0.3608 & -0.0001 & 2 \\
\hline 标定组 2 & 6 & 0.8411 & 0.00005 & 3 \\
\hline
\end{tabular}

表 4 非正交组 IMU 标定后残差

\begin{tabular}{ccccc}
\hline 组别 & 陀螺标号 & 安装偏差 $\left({ }^{\circ}\right)$ & $\begin{array}{c}\text { 刻度因子 } \\
\text { 估计误差 }\end{array}$ & $\begin{array}{c}\text { 陀螺常值漂移 } \\
(\% / h)\end{array}$ \\
\hline 标定组 1 & 1 & 0.0045 & 0.000034 & -0.0027 \\
标定组 1 & 3 & 0.0015 & -0.000032 & 0.0049 \\
标定组 1 & 4 & 0.0024 & -0.000033 & 0.0045 \\
标定组 2 & 2 & 0.0033 & 0.000002 & 0.0065 \\
标定组 2 & 5 & 0.0043 & -0.000023 & -0.0015 \\
标定组 2 & 6 & 0.0063 & 0.000040 & -0.0116 \\
\hline
\end{tabular}

表 $50.1^{\circ}$ 偏差下最小二乘和扩展卡尔曼滤波结果比对

\begin{tabular}{cccc}
\hline 算法名称 & 陀螺 1 误差 $\left(^{\circ}\right)$ & 陀螺 2 误差 $\left(^{\circ}\right)$ & 陀螺 3 误差 $\left(^{\circ}\right)$ \\
\hline 最小二乘 & 0.0030 & 0.0034 & 0.0035 \\
扩展卡尔曼滤波 & 0.0015 & 0.0040 & 0.0031 \\
\hline
\end{tabular}

表 $60.5^{\circ}$ 偏差下最小二乘和扩展卡尔曼滤波结果比对

\begin{tabular}{cccc}
\hline 算法名称 & 陀螺 1 误差 $\left(^{\circ}\right)$ & 陀螺 2 误差 $\left({ }^{\circ}\right)$ & 陀螺 3 误差 $\left({ }^{\circ}\right)$ \\
\hline 最小二乘 & 0.0044 & 0.0061 & 0.0051 \\
扩展卡尔曼滤波 & 0.0036 & 0.0077 & 0.0046 \\
\hline
\end{tabular}

及 IMU 上自身携带的立方镜, 计算出 IMU 在转台上 的安装矩阵、转台坐标系与地理坐标系的转换矩阵. 远程终端对转台控制柜发送采样命令, 获得转台框 架角数据, 根据转台框架角数据、转台坐标系与地理 坐标系的转换矩阵、当地地理纬度以及试验进行的累 积时间, 计算出惯性姿态数据. GNC 控制器根据 IMU 的测量数据以及惯性姿态数据, 进行陀螺标定计算, 获得 IMU 在转台上的安装矩阵, 其中 GNC 控制器中 装订的安装阵为引入误差后的安装阵, 以使得各陀 螺敏感轴存在一定安装偏差. 最终标定结果与光学 标定结果比对, 从而确定地面试验的有效性及标定 精度.

瞬时凝固惯性系 $o_{i} x_{i} y_{i} z_{i}$ 定义为: 原点 $o_{i}$ 位于地心; $o_{i} x_{i}$ 轴在试验开始时刻的平赤道面内, 指向 IMU 所在 的子午线与平赤道面的交点; $o_{i} z_{i}$ 轴垂直于试验开始时 刻的平赤道面, 指向地球自转方向; 三轴构成右手系. 


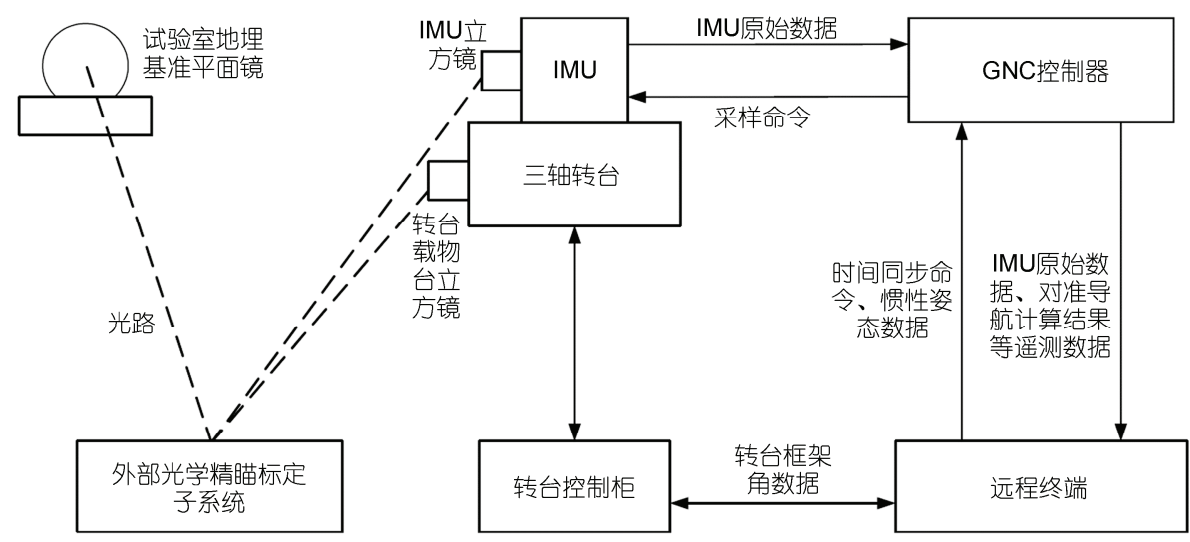

图 3 转台试验系统组成

地心地固系 $o_{o} x_{o} y_{o} z_{o}$ 定义为: 原点 $o_{o}$ 位于地心; $o_{o} x_{o}$ 轴在平赤道面内, 指向 IMU 所在的子午线与平赤 道面的交点; $o_{i} z_{i}$ 轴垂直于试验开始时刻的平赤道面, 指向地球自转方向; 三轴构成右手系, 并与地球固连. 在试验开始时刻, 地心地固系与瞬时凝固惯性系重 合.

远程终端中惯性姿态的计算为

$$
C_{t i}=C_{t t 0}\left(\phi_{i}, \phi_{m}, \phi_{o}\right) \times C_{t 0 e} \times C\left(\phi_{e}\right) \times C z\left(\omega_{e} t\right),
$$

其中, $\phi_{e}$ 为当地地理纬度, $\omega_{e}$ 为地球自转角速度, $t$ 为 从试验开始时刻累积的时间, $\phi_{o}, \phi_{m}, \phi_{i}$ 分别为三轴转 台的外、中、内 3 个框架角, $C_{t t 0}\left(\phi_{i}, \phi_{m}, \phi_{o}\right)$ 表示根据框 架角计算出的从 $t_{0}$ 时刻转台系到 $t$ 时刻转台系的转换 矩阵, $C\left(\phi_{e}\right)$ 表示从地心地固坐标系到当地地理坐标 系的转换矩阵.

\section{3 在轨飞行结果评价}

根据飞行程序安排, 月地高速再入返回飞行器 分别于 2014 年 10 月 25 日、 10 月 28 日和 10 月 29 日进行了 3 次 IMU 在轨标定. 首次标定初值采用地 面精测结果, 后 2 次标定均将前一次标定结果作为初 值进行迭代计算, 计算标定结果相对于初始值的变 化量作为标定误差, 表 8 中给出了 3 次标定后的安装 偏差的残差.

由表 8 标定结果可知, 受飞行器发射振动、在轨 热变形以及微重力等不确定性因素影响, 地面精测 的陀螺安装偏差与首次标定结果误差较大, 随着后 续 2 次的迭代标定过程, 误差逐步收玫. 为进一步验 证标定的安装偏差准确度, 分别采用地面精测的安 装矩阵和标定后的安装矩阵进行 $3000 \mathrm{~s}$ 导航计算,
并与在轨星敏感器 $3000 \mathrm{~s}$ 后的四元数进行比对.

由表 9 导航结果可知, 采用标定后安装矩阵和陀 螺常值漂移进行导航计算, $3000 \mathrm{~s}$ 导航角度误差与采 用地面精测值相比有较大提高.

\section{4 总结}

为了提高再入返回过程中导航精度, 探月工程 三期月地高速再入返回飞行器需要在返回前的有限

\section{表 7 转台半物理试验 IMU 标定后误差}

\begin{tabular}{cccc}
\hline 组别 & 陀螺标号 & 安装偏差 $\left(^{\circ}\right)$ & 标定后残差 $\left({ }^{\circ}\right)$ \\
\hline 标定组 & 1 & 0.1 & 0.0113 \\
标定组 1 & 2 & 0.2 & 0.0197 \\
标定组 1 & 3 & 0.3 & 0.0192 \\
标定组 2 & 4 & 0.1 & 0.0056 \\
标定组 2 & 5 & 0.2 & 0.0125 \\
标定组 2 & 6 & 0.3 & 0.0109 \\
\hline
\end{tabular}

\section{表 8 在轨 3 次 IMU 标定出的误差}

\begin{tabular}{cccc}
\hline 组别 & 第一次误差 $\left({ }^{\circ}\right)$ & 第二次误差 $\left(^{\circ}\right)$ & 第三次误差 $\left(^{\circ}\right)$ \\
\hline 陀螺 1 & 0.0473 & 0.0125 & 0.0054 \\
陀螺 2 & 0.0346 & 0.0141 & 0.0039 \\
陀螺 3 & 0.0252 & 0.0069 & 0.0059 \\
陀螺 4 & 0.0848 & 0.0068 & 0.0054 \\
陀螺 5 & 0.0335 & 0.0059 & 0.0057 \\
陀螺 6 & 0.0816 & 0.0148 & 0.0041 \\
\hline
\end{tabular}

\section{表 $93000 \mathrm{~s}$ 导航结果比对}

\begin{tabular}{cccc}
\hline 类别 & 姿态 $\phi$ 偏差 $\left(^{\circ}\right)$ & 姿态 $\theta$ 偏差 $\left(^{\circ}\right)$ & 姿态 $\psi$ 偏差 $\left(^{\circ}\right)$ \\
\hline 地面精测 & 0.0731 & 0.1235 & 0.0245 \\
在轨标定 & -0.0025 & -0.0010 & 0.0017 \\
\hline
\end{tabular}


时间内进行 2 套惯性测量单元组的安装偏差, 刻度因 子和常值漂移在轨标定. 根据这一特定任务需求, 本 文设计了一种基于最小二乘法的轻量化在轨陀螺标定 算法和地面试验验证方法, 能够在有限计算能力下同
时对多组陀螺进行在轨标定. 本方法具有对准精度高, 容错能力强, 故障数据易于剔除的特点. 探月三期月 地高速再入返回飞行器在轨成功地利用该方法实现了 2 套惯性导航平台的高精度初始姿态同步对准.

\section{参考文献}

1 Chaudhuri S K, Nandi P K. Transfer alignment for space vehicles launched form a moving base. Defense Sci J, 2005, 55: 245-252

2 王东升, 艾光涁, 吕善民, 等. 舰载平台式惯导系统的传递对准. 中国惯性技术学报, 2009, 17: 24-27

3 戴郡武, 李娟, 戴洪德, 等. 一种快速对准传递方法的误差模型研究. 宇航学报, 2009, 30: 942-946

4 Goshen M D, Bar I Y. Unified approach to inertial navigation system error modeling. AIAA Jf Guid Control Dynam, 2012, 15: 648-653

5 徐林, 李世玲, 屈新芬. 惯导导航系统传递对准技术综述. 信息与电子工程, 2010, 8: 633-640

6 夏家和, 秦永元, 赵长山. 基于测量矢量匹配的传递对准方法研究. 系统工程与电子技术, 2009, 31: 2946-2948

7 LiuX X, Xu X S, Liu Y T, et al. A fast and high-accuracy transfer alignment method between M/S INS for ship based on iterative calculation. Measurement, 2014, 51: 297-309

8 Dai H D, Dai S W, Cong Y C, et al. Rapid transfer alignment of laser SINS using quaternion based angular measurement. Optik Int J Light Elect Opt, 2013, 124: 4364-4368

9 Chattaraj S, Mukherjee A, Chaudhuri S K. Transfer alignment problem: Algorithms and design issues. Gyroscopy Navigation, 2013, 4: 130-146

10 Emily L B, Allan Y L. In-flight characterization of Cassini inertial reference units. AIAA Guidance, Navigation and Control Conference and Exhibit. Hilton Head. South Carolina, 2007

11 李骥, 张洪华, 赵宇, 等. 嫦娥三号着陆器的陀螺在轨标定. 中国科学: 技术科学, 2014, 44: 582-588

12 Jones D, Roberts C, Tarrant D, et al. Transfer alignment design and evaluation environment. In: IEEE Proceedings of Aerospace Conrol Systems. Palm, 1993. 753-757

13 Shortelle K J, Graham W R, Rabourn C. F-16 flight tests of a rapid transfer alignment procedure. In: IEEE Position Location and Navigation Symposium. Palm, 1998. 379-386

14 You J C, Qin Y Y, Xia J H, et al. Transfer alignment design and experiment evaluation of the SINS for GMLRS artillery rocket. In: Sixth International Symposium on Precision Engineering Measurements and Instrumentation, Hangzhou, 2010. 7544M1-7544M8 


\title{
Research and implementation of on-orbit self-calibration for gyroscope of circumlunar return and reentry spacecraft
}

\author{
YU Dan ${ }^{1}$, DONG WenQiang ${ }^{1,2}$, WANG Yong ${ }^{1}$, ZHENG YongJie $^{1}$, YANG Ming ${ }^{1,2}$, ZHANG Zhao $^{1,2}$, \\ ZHANG GuoFeng $^{1} \&$ WANG ZhiYi ${ }^{1}$ \\ ${ }^{1}$ Beijing Institute of Control Engineering, Beijing 100190, China; \\ ${ }^{2}$ Science and Technology on Space Intelligent Control Laboratory, Beijing 100190, China
}

For the problems of lightening design of auto-alignment for strapdown inertial navigation platform included non-orthogonal gyroscope, an error model of non-orthogonal gyros is established in this paper, and we propose a lightweight calibration algorithm based on least square method and a ground test verification method, which achieved high accuracy calibration on-orbit within a limited amount of computing capacity.The ground mathematic simulation and the results of test validation show that our method has the similar accuracy to extended kalman filter, but the computational complexity is much less than EKF. On this basis, we achieved synchronous fault tolerant and calibration for multi-sets, multi-systems gyros on-orbit by logic design of calibration algorithm. This algorithm has already been used successfully in actual flight of circumlunar return and reentry spacecraft of 3rd phase of China lunar exploration program, and thus achieved initial attitude alignment of two-sets of inertial navigating platforms with a high degree of accuracy.

3rd phase of China lunar exploration program, circumlunar free return and reentry spacecraft, inertial measurement unit, lightweight calibration algorithm, self-calibration

doi: 10.1360/N092014-00481 